\title{
Holographic Renormalization and Ward Identities with the Hamilton-Jacobi Method ${ }^{\star}$
}

\author{
Dario Martelli ${ }^{\mathrm{a}}$ and Wolfgang Mück ${ }^{\mathrm{b}}$ \\ ${ }^{a}$ Queen Mary College, University of London, Mile End Road, London E1 4NS, UK \\ ${ }^{\mathrm{b}}$ Dipartimento di Scienze Fisiche, Università di Napoli "Federico II", Via Cintia, \\ 80126 Napoli, Italy
}

\begin{abstract}
A systematic procedure for performing holographic renormalization, which makes use of the Hamilton-Jacobi method, is proposed and applied to a bulk theory of gravity interacting with a scalar field and a $U(1)$ gauge field in the Stückelberg formalism. We describe how the power divergences are obtained as solutions of a set of "descent equations" stemming from the radial Hamiltonian constraint of the theory. In addition, we isolate the logarithmic divergences, which are closely related to anomalies. The method allows to determine also the exact one-point functions of the dual field theory. Using the other Hamiltonian constraints of the bulk theory, we derive the Ward identities for diffeomorphisms and gauge invariance. In particular, we demonstrate the breaking of $U(1)_{R}$ current conservation, recovering the holographic chiral anomaly recently discussed in hep-th/0112119 and hep-th/0202056.
\end{abstract}

Key words: AdS/CFT Correspondence, Renormalization and Regularization

\section{Introduction and Summary}

During the past four years, we have learned much from the AdS/CFT correspondence $[1,2,3]$ about conformal field theories (CFTs). The basic notion of the AdS/CFT correspondence is that a gravitational theory (such as String Theory) living on $(d+1)$-dimensional anti-de Sitter (AdS) space (the bulk space) is dual to a CFT living on its conformal boundary. More generally,

‡ Preprint numbers: hep-th/0205061, QMUL-PH-02-11, DSF-11-2002

Email addresses: d.martelli@qmul.ac.uk (Dario Martelli), mueck@na.infn.it (Wolfgang Mück).

Preprint submitted to Elsevier Science 30 October 2018 
asymptotically AdS domain wall solutions of $(d+1)$-dimensional gravity coupled to certain matter fields are the duals of deformations of CFTs either by the addition of relevant operators to the CFT Lagrangian or by the choice of a non-conformal ground state involving vev's of certain operators. Using a precise recipe one is able to obtain the correlation functions of these (deformed) CFTs from the dynamics of the bulk theory. The most important quantity is the on-shell action of the bulk theory, which, after suitable regularization and renormalization, is identified with the generating functional of the boundary field theory. For a recent exposition of the AdS/CFT correspondence including an extensive list of references, we refer the reader to the lecture notes [4].

The occurrence of divergences in the bulk on-shell action was noted already in the earliest AdS/CFT calculations $[2,3,5,6]$. The first divergence to be explicitly removed from the on-shell action by adding a counterterm was the boundary volume divergence in the context of pure gravity on an AdS background [7]. Soon after, and still for the case of pure gravity on an AdS background, the general structure of the divergent terms and the relation of the logarithmic divergence to the conformal anomaly of the boundary CFT was discussed in [8]. The addition of counterterms also provided a way to give a meaning to the notion of energy in asymptotically AdS spaces in terms of the renormalized Brown-York stress energy tensor [9] without the need of reference spaces $[10,11,12]^{1}$

A systematic development of holographic renormalization for bulk gravity coupled to scalar fields was first given in [15]. This method, which we shall refer to as the standard method of holographic renormalization, involves the cancellation of all cut-off related divergences from the bulk on-shell action by the addition of counterterms on a cut-off boundary hypersurface and the subsequent removal of the cut-off. The counterterms removing power divergences are fully covariant expressions of fields living at the cut-off boundary, whereas logarithmic counterterms depend also explicitly on the cut-off. This dependene breaks some bulk diffeomorphisms and yields, e.g., the trace anomaly [8]. Most recently, the standard method was summarized very clearly by Bianchi, Freedman and Skenderis (BFS) [16] (see also the lecture notes by Skenderis [17]) and applied to domain wall bulk geometries dual to deformed CFTs, where also bulk gauge fields were considered. Holographic renormalization has been used in the calculation of two-point functions in deformed CFTs [18].

In a parallel development, de Boer, Verlinde and Verlinde (dBVV) [19] advocated the Hamilton-Jacobi (HJ) approach in order to separate terms in the bulk on-shell action, which can be written as local functionals of the boundary data. The remaining, presumably non-local, expression was identified with

1 For example, compare the treatments of the AdS-Schwarzschild black hole using pure AdS as reference space [13] and using counterterms [14]. 
the generating functional of a boundary field theory. This approach does not correspond to the standard one, in particular, because dBVV's boundary field theory lives on the cut-off boundary, and because the generating functional still contains logarithmic divergences. Nevertheless, dBVV's method remains intriguing for its simplicity - it yields formally the correct gravitational anomalies and provides a remarkably simple bulk description of the renormalization group flow in deformed CFTs. A first attempt to use the Hamilton-Jacobi method for performing holographic renormalization appeared in [20], where a list of references to further studies of dBVV's method can be found.

Despite its simplicity, the use of dBVV's method to perform holographic renormalization does no appear to be very popular. The main drawbacks in its original formulation seem to be the non-uniqueness of the solutions for the local terms to be used as counterterms and the failure to obtain the logarithmic counterterms. It is our intention in this paper to overcome these difficulties by explaining exactly how the ambiguities can be removed from dBVV's method and how logarithmic counterterms are obtained. The main steps of our approach, which we shall call the HJ method of holographic renormalization, will be as follows. In the first step, we shall use dBVV's method to determine the counterterms for the power divergences. Ambiguities in the descent equations are removed at the top level by comparison with simple and known free field calculations. In the second step, we continue to analyze the hamiltonian constraint with two important results. First, we prove that all power divergences have been removed by the first step, and, second, we obtain explicitly the logarithmic divergence and remove it. Hence, we prove that the HJ method qualifies as a consistent procedure for performing holographic renormalization. We point out that the difference between the standard method and the HJ method does not stem from using different renormalization schemes, but regards only the procedure in which the (exactly identical) counterterms are obtained. In both methods a choice of renormalization scheme is reflected in the possibility of adding finite counterterms.

A very interesting aspect of the HJ formalism is the way holographic Ward identities emerge. In fact, local symmetries of the bulk theory are encoded in hamiltonian constraints for the boundary data. Exploiting the fact that the on-shell action is identified with the field theory generating functional, these can be naturally interpreted as Ward identities [21,22]. The hamiltonian constraint associated with radial evolution can be seen as the Ward identity for conformal symmetry, whose anomalous contributions are related to the logarithmic divergences of the on-shell action [8]. Similarly, we will see that logarithmic terms are also responsible for an anomalous term in the current Ward identity.

Let us give an outline of the rest of the paper and summarize our results. In Sec. 2 we shall briefly review the standard method of holographic renormal- 
ization in order to familiarize the reader with the issue and the complexity of the method, and to allow a direct comparison with the approach that we will propose. We will then turn to the HJ method with the purpose of performing the holographic renormalization. The method will be compared to the standard method at appropriate points throughout the paper. For simplicity, in the first part of the paper we shall confine our attention to a bulk gravity theory coupled to a single scalar field. In Sec. 3 we focus on the procedure of regularization and renormalization of the bulk on-shell action. This is carried out solving the hamiltonian constraint for radial evolution in a recursive fashion. Following dBVV, we start with a general ansatz for the leading local terms of the on-shell action, which should be covariant and contain all power divergences. The constraint will then fix all of them, provided some little input is given for the lowest levels (Sec. 3.1). As was already discussed in $[19,20]$, there might be some obstruction to the solution of the constraint, which is closely related to the occurrence of a conformal anomaly. We will make this observation more precise and relate the anomaly to the logarithmic divergences of the on-shell action, which we can explicitly determine and remove. This is carried out in Sec. 3.2, where we also prove that all divergences have been removed.

In Sec. 4.1, we shall discuss how to determine exact one-point functions in our method. By "exact" we mean one-point functions in the presence of sources turned on, thus, in principle, encoding all higher point functions of the field theory. This is one of the main results of BFS's approach, and any alternative method should not fall short of it. Our results will also explicitly demonstrate the scheme dependence of local terms in the exact one-point functions. As a further application, we consider one-point functions in bulk backgrounds, which are called holographic renormalization group flows (Sec. 4.2). The difference between operator and vev flows, which describe deformations of the dual CFT by either operator insertion or a non-zero vev, respectively, will become explicit. Moreover, the most natural choice of counterterms will correspond to a supersymmetric renormalization scheme.

In Sec. 5 we will include a $U(1)$ gauge field in our treatment, which should correspond to the $R$-symmetry current of the dual field theory [23,16]. A repetition of the arguments previously explained will allow us to show how to solve for the on-shell action up to level four (with some simplifications) and to obtain new contributions to the conformal anomaly. We will obtain some one-point functions for the new fields and, in particular, we will give a full holographic derivation of the complete Ward identities, recovering also the chiral anomaly recently discussed in [16], [24] and [25]. 


\section{Holographic Renormalization - Standard Approach}

To start, let us summarize the standard method of holographic renormalization as described by BFS [16]. For later convenience, we shall use a bulk metric of the form

$$
d s^{2}=d r^{2}+g_{i j}(x, r) d x^{i} d x^{j},
$$

where $i, j=1 \ldots d$. The metric (1) is related to the Fefferman-Graham metric for asymptotically AdS spaces [26] by a simple change of variables. The asymptotic region is $r \rightarrow \infty$, where the bulk approaches AdS spacetime with characteristic length $l$, i.e.,

$$
g_{i j}(x, r) \rightarrow \mathrm{e}^{2 r / l} \hat{g}_{i j}(x) .
$$

The standard method starts by solving the equations of motion in the asymptotically AdS region, which yields the bulk fields in the form of asymptotic series like ${ }^{2}$

$$
\begin{aligned}
F(x, r)= & \mathrm{e}^{\lambda_{1} r / l}\left[f_{0}(x)+\mathrm{e}^{r / l} f_{1}(x)+\mathrm{e}^{2 r / l} f_{2}(x)+\cdots\right] \\
& +\mathrm{e}^{\lambda_{2} r / l}\left[\tilde{f}_{0}(x)+\mathrm{e}^{r / l} \tilde{f}_{1}(x)+\mathrm{e}^{2 r / l} \tilde{f}_{2}(x)+\cdots\right],
\end{aligned}
$$

consisting of the two independent asymptotic series solutions of the equations of motion. These series expansions are similar to the Fefferman-Graham expansion of the metric for asymptotically AdS spaces [26] in pure gravity.

The assumption that the bulk space be asymptotically AdS implies that the fields are asymptotically free, and thus the coefficients $\lambda_{1}$ and $\lambda_{2}$ are related to the asymptotic mass of the field $F$, whereas $f_{0}$ and $\tilde{f}_{0}$ are independent integration constants. Without loss of generality, we shall assume that $\lambda_{1}>\lambda_{2}$, so that the first line in eqn. (3) represents the leading series solution. Then, $f_{0}$ is called the source of $F$. The coefficients $f_{1}, f_{2}$, etc., and similarly, $\tilde{f}_{1}, \tilde{f}_{2}$, etc., are obtained by recursive analysis of the bulk field equations in the asymptotic region and depend locally on the $f_{0}$ and $\tilde{f}_{0}$ of all fields, respectively. In the dual boundary field theory, the source $f_{0}$ is the generating current of the operator that is dual to the bulk field $F$, while $\tilde{f}_{0}$ is related to the vev of this operator (see Sec. (4.1)).

2 Although this form is not generic, it suffices to describe the standard method. The expansion (3) will in general contain not only powers of $e^{r / l}$ and the logarithmic terms described later, because bulk interactions and generic values of the conformal dimensions will generate non-integer exponents. The literature on the standard method $[15,16]$ starts with expansions of the form (3), because it covers most interesting cases, where conformal dimensions are integer or half-integer. However, there is no obstruction in principle to apply the method to other cases [27]. 
For example, for the metric, $g_{i j}$, the coefficients are $\lambda_{1}=2$ and $\lambda_{2}=2-d$. In the case of a scalar field, $\phi$, of bulk mass $m$, they are $\lambda_{1}=-(d / 2-\lambda)$ and $\lambda_{2}=-(d / 2+\lambda)$, where $\lambda=\sqrt{d^{2} / 4+m^{2} l^{2}}$. The bulk field $\phi$ is the dual of a scalar operator with conformal dimension $\Delta=d / 2+\lambda$. We shall consider only matter fields dual to relevant operators, for which $\lambda_{1}<0$, so that they tend to zero in the asymptotic region. ${ }^{3}$ For even values of $d$, there are logarithmic terms in the leading series for the metric (proportional not to an exponential of $r$, but to $r)$, starting with $r \times \exp [(2-d) r / l]$. Similarly, logarithmic terms occur in the leading series of scalar fields for integer $\lambda$.

Having obtained the asymptotic form of the bulk fields, one proceeds to calculate the regulated on-shell action, which is the on-shell action for a bulk spacetime with a cut-off boundary at $r=\rho$,

$$
S_{\mathrm{reg}}\left[f_{0}, \rho\right]=\int d^{d} x \sqrt{g_{0}}\left[\mathrm{e}^{\nu_{0} \rho / l} a_{0}\left(f_{0}\right)+\mathrm{e}^{\nu_{1} \rho / l} a_{1}\left(f_{0}\right)+\cdots+\frac{\rho}{l} \bar{a}\left(f_{0}\right)+O(1)\right],
$$

where the $\nu_{k}$ are positive numbers. There are a finite number of divergent terms in the limit $\rho \rightarrow \infty$, which comprise the power divergences with coefficients $a_{k}$ and a logarithmic divergence with a coefficient $\bar{a}$ that is related to the appearance of logarithmic terms in the expansion (3). The coefficients $a_{k}$ and $\bar{a}$ are local functions of the coefficients $f_{0}$ of the leading series of the bulk fields and of their derivatives.

The method of holographic renormalization proceeds now as follows. First, the series (3) are inverted for all fields and solved for the sources $f_{0}(x)$ as functionals of the $F(x, r)$. Second, the $f_{0}$ thus obtained are substituted into eqn. (4), which yields the divergent terms of the on-shell action in terms of the fields $F(x, \rho)$ living at the cut-off boundary. Finally, these terms are subtracted from $S_{\text {reg }}$ (using counterterms), and in the remaining expression the limit $\rho \rightarrow \infty$ is taken. The finite result (expressed again as a functional of the sources $f_{0}$ ) is the renormalized on-shell action to be identified with the generating functional of the boundary field theory.

BFS's method is clear and rigorous. The four steps of the procedure - the asymptotic expansion of the fields, the recursive determination of the coefficients, the inversion of the asymptotic series and the substitution of the inverted series into the on-shell action - form an algorithm that uniquely yields all counterterms needed to cancel the $\rho \rightarrow \infty$ divergences of the bulk on-shell action. Further finite counterterms could be added and correspond to a certain choice of renormalization scheme. One should notice the importance of using covariant counterterms, hence the need to invert the asymptotic series solutions of the fields. Covariance of the counterterms implies the validity of the Ward identity of diffeomorphisms of the boundary field theory. In fact,

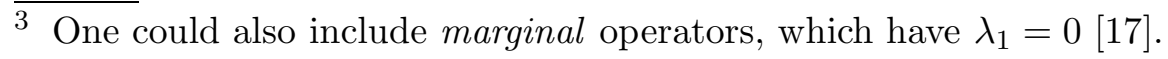


the counterterms, when expressed in terms of $f_{0}$ and $\tilde{f}_{0}$, contain finite terms that affect the renormalized on-shell action and do not correspond to a choice of renormalization scheme, i.e., they contribute to the physical results.

It is an interesting question whether there exists a method, which determines the counterterms directly at the cut-off boundary without performing the asymptotic expansion and its inversion. Such a method is provided by the Hamilton-Jacobi approach, which we shall turn to in the next section.

\section{Holographic Renormalization - Hamilton-Jacobi Approach}

As mentioned in the previous sections, we propose to use the HJ method advocated for the AdS/CFT correspondence by dBVV in order to determine the counterterms. In this section, we shall describe in detail how the counterterms are obtained, starting with the power divergences in Sec. 3.1 and discussing the logarithmic divergences in Sec. 3.2. The relation between logarithmic divergences and conformal anomalies will become explicit, a fact that has been used by Henningson and Skenderis [8] in the derivation of the conformal anomaly.

In order to keep the presentation simple and to concentrate on the main steps of the method, we consider in this section the action of gravity coupled to a single scalar field ${ }^{4}$

$$
S=\int d^{d+1} x \sqrt{\tilde{g}}\left[-\frac{1}{4} \tilde{R}+\frac{1}{2} \tilde{g}^{\mu \nu} \partial_{\mu} \phi \partial_{\nu} \phi+V(\phi)\right]+\frac{1}{2} \int d^{d} x \sqrt{g} H
$$

The necessary formulae for the HJ method are summarized in the appendix, and the vector sector should be omitted here. We shall include the gauge fields in Sec. 5 .

Before plunging into the details, let us briefly compare the HJ approach with the standard method of holographic renormalization. In the HJ approach, the analysis is carried out directly at the cut-off boundary, so that an asymptotic expansion and its inversion are not necessary, except for the leading order term determined by the behaviour of the free fields. The recursive determination of the series coefficients from the field equations is replaced by the recursive solution of a set of descent equations. In order to determine where to stop (if the system does not break down by itself), a simple power counting using the leading behaviour of the fields is sufficient. The descent equations are derived from one equation only, which is the hamiltonian constraint, using the independence of the boundary conditions in order to split the constraint into

4 Details of our notation are given in the appendix. For the more general case of several scalars, see [20]. 
functionally independent terms. One should expect ambiguities, because this equation does not contain all information about the field dynamics. In fact, the descent equations do not yield a unique solution for the counterterms, but, as we shall see, the ambiguities appear right at the top of the descent equations and can be easily removed with little input from the (known) results for free fields. The subsequent analysis of the descent equations yields a unique answer for the divergent counterterms. As a very nice feature, the other constraint equations translate into Ward identities of the dual field theory.

\subsection{Power Divergences}

In this section, we shall briefly outline how the power divergences of the onshell action are obtained using the method of dBVV. In order to separate the power divergences, the on-shell action is split up as

$$
S=S_{[0]}+S_{[2]}+\cdots+S_{[2 n]}+\Gamma
$$

where we have denoted power divergent terms by $S_{[2 k]}, k=0 \ldots n$. According to eqn. (A.21), the momentum $\pi$ naturally splits into

$$
\pi=\pi_{[0]}+\pi_{[2]}+\cdots+\pi_{[2 n]}+\pi_{\Gamma}
$$

and similarly for $q_{j}^{i}$. We shall make the following premises regarding the counterterms $S_{[2 k]}$.

(1) The counterterms are covariant (and gauge invariant, if vector fields are involved) local expressions in terms of the metric $g_{i j}$ and the field $\phi$ at the cut-off boundary as well as their derivatives.

(2) The term $S_{[2 k]}$ contains exactly $k$ inverse metrics. (This is an arbitrary assumption, but turns out to be very useful for the bookkeeping.)

(3) The counterterms should completely contain the power divergences of $S$.

(4) The counterterms must be universal, i.e., they must contain the power divergences of $S$ for any asymptotically AdS solution of the bulk equations of motion.

The premises 1 and 2 imply that we can write

$$
\begin{aligned}
& S_{[0]}=\int d^{d} x \sqrt{g} U(\phi), \\
& S_{[2]}=\int d^{d} x \sqrt{g}\left[\frac{1}{2} M(\phi) g^{i j} \partial_{i} \phi \partial_{j} \phi+\Phi(\phi) R\right], \text { etc. }
\end{aligned}
$$

The number $n$ of power divergent terms is dictated by premise 3: By power counting using the leading asymptotic behaviour of the fields for $r \rightarrow \infty$ [cf. eqn. (3)], one can determine which covariant boundary integrals are generically divergent. All of these have to be included. For example, an $S_{[2 k]}$ without 
derivatives of matter fields behaves generically like $\exp [(d-2 k) \rho / l]$ from the metric contributions. Thus, we must include all of these with $k<d / 2$. In eqn. (6), $\Gamma$ may contain a logarithmic divergence, but shall be regarded as finite for the power counting. Finite local terms can be arbitrarily shifted between $\Gamma$ and the counterterms. This reflects the usual ambiguity of choosing a renormalization scheme.

The main point of dBVV's method is to analyze the Hamiltonian constraint [eqn. (A.17)] by splitting it into a derivative expansion, ${ }^{5}$

$$
\mathcal{H}=\mathcal{H}_{[0]}+\mathcal{H}_{[2]}+\cdots+\mathcal{H}_{[4 n]}+\mathcal{H}_{\Gamma}=0
$$

where $\mathcal{H}_{[2 k]}$ denotes those terms in $\mathcal{H}$ that stem only from the counterterms and contain a total of $k$ inverse metrics. Notice that eqn. (10) only re-writes the hamiltonian $\mathcal{H}$ in a form, which makes the contributions from the various counterterms explicit. By construction, a certain number of $\mathcal{H}_{[2 k]}$ are asymptotically stronger than $\mathcal{H}_{\Gamma}$, and these can be found by simple power counting. One should now try to solve the constraint separately for these (stronger) $\mathcal{H}_{[2 k]}$, starting with $\mathcal{H}_{[0]}$, which leads to a system of descent equations. This approach is justified by the universality premise.

Let us demonstrate the procedure by considering the level 0 and level 2 terms of the Hamiltonian. For more details, see $[19,20]$. The level 0 descent equation is given by

$$
\mathcal{H}_{[0]}=-\frac{d}{d-1} U^{2}+\frac{1}{2}\left(U^{\prime}\right)^{2}-V=0 .
$$

This equation is regarded as a functional equation for $U(\phi)$, where $V(\phi)$ is given. Solving eqn. (11) is impossible in closed form for a generic potential $V(\phi)$ (see $[30,31]$ for a numerical analysis). However, we are interested in removing the $\rho \rightarrow \infty$ divergences, and thus an analysis in the asymptotic region is sufficient. In fact, because the leading behaviour of a relevant scalar is $\phi(x, r) \sim \phi_{0}(x) \mathrm{e}^{\lambda_{1} r}$ with $\lambda_{1}<0$ [cf. eqn. (3)], for any finite, but otherwise arbitrary value of the source $\phi_{0}$, we can choose the cut-off $\rho$ sufficiently large such as to bring the Dirichlet value $\phi(x, \rho)$ within an arbitrarily small neighbourhood of the fixed point, $\phi=0$. The same is true for any number of relevant matter fields. Hence, it is sufficient to solve $\mathcal{H}_{[0]}=0$ close the fixed point. For this purpose, we expand it into a Taylor series. ${ }^{6}$ Thus, we write

5 Such kinds of expansions were proposed previously in the literature as a method for solving the Hamilton-Jacobi equations of General Relativity in the presence of some matter fields $[28,29]$.

${ }^{6}$ Note that this is not equivalent to a large $\rho$ expansion of the descent equations, because it does not take into account the different large $\rho$ behaviours of different scalars [27]. 
$V(\phi)$ as

$$
V=-\frac{d(d-1)}{4 l^{2}}+\frac{1}{2} m^{2} \phi^{2}+\frac{1}{3 !} v_{3} \phi^{3}+\frac{1}{4 !} v_{4} \phi^{4}+O\left(\phi^{5}\right),
$$

where the constant part represents the negative cosmological constant, and $m$ is the bulk mass parameter for $\phi$. Of course, this assumes that the potential $V$ can be expanded in a Taylor series, which we shall assume to be true. Similarly, we expand $U$ as

$$
U=u_{0}+u_{1} \phi+\frac{1}{2} u_{2} \phi^{2}+\frac{1}{3 !} u_{3} \phi^{3}+\frac{1}{4 !} u_{4} \phi^{4}+O\left(\phi^{5}\right) .
$$

Now, we obtain from eqn. (11) a set of coupled equations, from which the coefficients $u_{k}$ should be recursively determined. It turns out that there is no unique solution, but input from the (known) free field behaviour will fix the ambiguities. First, we see that the action (5) does not contain terms, which are linear in $\phi$. Thus, there cannot be divergent terms linear in $\phi$, and we shall set

$$
u_{1}=0 .
$$

This solves the term of order $\phi$ of eqn. (11). Then, we find from the constant term

$$
\left(u_{0}\right)^{2}=\frac{(d-1)^{2}}{4 l^{2}} .
$$

Here, we must choose a sign, and in order to choose the correct one, let us compare with a simple known case. Liu and Tseytlin [7] determined this leading counterterm as

$$
u_{0}=-\frac{d-1}{2 l},
$$

which cancels the volume divergence of the on-shell action for pure gravity [7]. This is easy to verify for a bulk AdS spacetime with a flat cut-off boundary. Next, the quadratic term of eqn. (11) yields

$$
\left(u_{2}\right)^{2}+\frac{d}{l} u_{2}-m^{2}=0,
$$

where eqns. (14) and (16) have been used. Again, the solution is not unique, and we must resort to the free scalar field in AdS background, whose on-shell action is

$$
S=\frac{1}{2} \int d^{d} x \sqrt{g} \phi \partial_{r} \phi,
$$

and whose leading behaviour (obtained from the free equation of motion) is

$$
\phi(x, r) \sim \mathrm{e}^{(-d / 2+\lambda) r / l} \hat{\phi}(x)
$$

with $\lambda=\sqrt{d^{2} / 4+m^{2} l^{2}}$. Hence, the leading behaviour of the on-shell action (18) is

$$
S \sim \frac{1}{2 l} \int d^{d} x \sqrt{g} \phi^{2}\left(-\frac{d}{2}+\lambda\right) .
$$


This tells us that the correct choice amongst the solutions of eqn. (17) is

$$
u_{2}=\frac{1}{l}\left(-\frac{d}{2}+\lambda\right) \text {. }
$$

Continuing with the cubic term of eqn. (11), we obtain

$$
v_{3}=\frac{u_{3}}{l}\left(3 \lambda-\frac{d}{2}\right)
$$

so that, for $\lambda \neq d / 6$, we uniquely find

$$
u_{3}=\frac{2 l}{6 \lambda-d} v_{3} .
$$

In contrast, for $\lambda=d / 6$, the coefficient $u_{3}$ remains undetermined, and we have a remaining term,

$$
\mathcal{H}_{\text {rem }}=-\frac{1}{6} v_{3} \phi^{3}+O\left(\phi^{4}\right) .
$$

We shall postpone the discussion of $\mathcal{H}_{\text {rem }}$ until Sec. 3.2. The fact that $u_{3}$ remains undetermined is a sign of the renormalization scheme dependence. In fact,

$$
\int d^{d} x \sqrt{g} \phi^{3} \sim \mathrm{e}^{d r / l}\left[\mathrm{e}^{(-d / 2+\lambda) r / l}\right]^{3}=1 \quad \text { for } \lambda=d / 6,
$$

so that the undetermined counterterm is finite. More generally, a breakdown of the level 0 descent equation will occur, if

$$
\lambda=\frac{(k-2) d}{2 k}
$$

for some integer $k>2$ [31]. In this case, the coefficient $u_{k}$ remains undetermined, and the boundary integral of $\phi^{k}$ is finite in the $\rho \rightarrow \infty$ limit. A relation similar to eqn. (26) can be found also at the higher levels.

For later use, let us also consider the $\phi^{4}$ term of the constraint (11), which becomes

$$
\frac{u_{4}}{24 l}(4 \lambda-d)-\frac{v_{4}}{24}-\frac{d}{4(d-1) l^{2}}\left(\frac{d}{2}-\lambda\right)^{2}+\frac{l^{2} v_{3}^{2}}{2(6 \lambda-d)^{2}}=0
$$

after the coefficients $u_{0}, u_{1}, u_{2}$ and $u_{3}$ have been inserted. Clearly, if $\lambda \neq d / 4$, eqn. (27) determines $u_{4}$ uniquely, but, if $\lambda=d / 4$, the descent equation breaks and there is a remainder

$$
\mathcal{H}_{\text {rem }}=\left(-\frac{v_{4}}{24}-\frac{d^{3}}{64(d-1) l^{2}}+\frac{2 l^{2} v_{3}^{2}}{d^{2}}\right) \phi^{4}+O\left(\phi^{5}\right) .
$$

It turns out that the relevant contribution to $\mathcal{H}_{\text {rem }}$ might be zero in particular cases. An example is the potential responsible for the GPPZ flow [32], where 
$d=4$ and $\lambda=d / 4=1$. The breakdown occurs for $k=4$, but we have $v_{4}=-8 / l^{2}$ and $v_{3}=0$, so that there is no $\phi^{4}$ contribution to $\mathcal{H}_{\text {rem }}$.

It is useful to pause for a moment and reflect on some details of what we have done so far. The idea for finding the power divergent terms is to write down the most general set of covariant local counterterms up to the necessary level and solve for them by asking that they satisfy the hamiltonian constraint for arbitrary and independent values of boundary conditions (Dirichlet values at the cut-off boundary) of the fields. One might object that this assumes that all power divergences can be cancelled by covariant local counterterms, whereas, in the standard method, this is shown by explicit construction. At this stage of the procedure, we do not have any proof that the procedure applied so far yields all power divergences, but this will be proven in Sec. 3.2.

The origin of the ambiguities we have met becomes clear when considering the hamilton equations of motion, e.g., eqns. (A.13) and (A.14). The choice of sign of $u_{0}$ reflects the fact that one can choose both, $r \rightarrow \infty$ and $r \rightarrow-\infty$, as the asymptotic region, and the choice in eqn. (16) corresponds to $r \rightarrow \infty$. Similarly, the two possible solutions of eqn. (17) describe, via eqn. (A.14), the two independent asymptotic behaviours of the scalar field. The counterterm must describe the leading one, because it is asymptotically strongest, and this is ensured by the choice in eqn. (21). It is now also clear that further ambiguities can appear neither for higher powers of $\phi$ nor at the higher levels. In fact, the hamilton equations of motion provide a one-to-one correspondence between the first few terms of the leading asymptotic series and the counterterms. Hence, since the leading asymptotic series solution is unique for any given sources, there is exactly one set of universal covariant counterterms.

Let us now turn to the level 2 equation, which reads

$$
\begin{aligned}
\mathcal{H}_{[2]} & =\left(-\frac{d-2}{d-1} U M+4 U \Phi^{\prime \prime}-\frac{1}{2} U^{\prime} M^{\prime}-\frac{1}{2}\right) \nabla_{i} \phi \nabla^{i} \phi \\
& +\left(4 U \Phi^{\prime}-U^{\prime} M\right) \nabla_{i} \nabla^{i} \phi \\
& +\left(-2 \frac{d-2}{d-1} \Phi U+U^{\prime} \Phi^{\prime}+\frac{1}{4}\right) R=0 .
\end{aligned}
$$

By the universality premise, functionally independent terms must vanish separately, and this yields the following three descent equations,

$$
\begin{aligned}
-\frac{d-2}{d-1} U M+4 U \Phi^{\prime \prime}-\frac{1}{2} U^{\prime} M^{\prime} & =\frac{1}{2} \\
4 U \Phi^{\prime}-U^{\prime} M & =0 \\
2 \frac{d-2}{d-1} \Phi U-U^{\prime} \Phi^{\prime} & =\frac{1}{4}
\end{aligned}
$$


These have been analyzed in [20], and the solutions are

$$
\Phi=\Phi_{0}+\Phi_{1} \phi+\frac{1}{2} \Phi_{2} \phi^{2}+O\left(\phi^{3}\right),
$$

with

$$
\begin{aligned}
& \Phi_{0}=-\frac{l}{4(d-2)} \quad \text { for } d \neq 2, \\
& \Phi_{1}=0 \\
& \Phi_{2}=-\frac{(d-2)(d-2 \lambda)}{2(d-1)(\lambda-1)} \Phi_{0} \quad \text { for } \lambda \neq 1,
\end{aligned}
$$

and

$$
M=\frac{l}{2(\lambda-1)}+O(\phi) \quad \text { for } \lambda \neq 1 .
$$

For $d=2$ and $\lambda=1$ the coefficients $\Phi_{0}, \Phi_{2}$ and $M_{0}$ remain undetermined, respectively, leaving a remainder

$$
\mathcal{H}_{\mathrm{rem}}= \begin{cases}-\frac{1}{2} \nabla^{i} \phi \nabla_{i} \phi-\frac{d-2}{8(d-1)} \phi^{2} R+O\left(\phi^{3}, R^{2}\right) & (\lambda=1), \\ \frac{1}{4} R+O\left(\phi, R^{2}\right) & (d=2) .\end{cases}
$$

Similarly, an analysis of the level 4 descent equations has been carried out in [20]. A breakdown at level 4 occurs, if $d=4$ or $\lambda=2$. For $d=4$, the unresolved remainder is

$$
\mathcal{H}_{\mathrm{rem}}=-\frac{l^{2}}{16}\left(\frac{1}{3} R^{2}-R_{i j} R^{i j}\right)+O\left(R^{3}\right) .
$$

The method can be extended recursively to any desired level, of course with a rapidly increasing amount of complexity. The highest level necessary to cancel the power divergent terms in $S$ is found by power counting. As discussed above, the method yields all covariant boundary terms in the on-shell action, which are divergent by power counting. These are interpreted as counterterms to be added, although it has not yet been shown that all power divergences of $S$ are obtained. It also gives information on possible scheme dependent finite terms, and one may choose to add also irrelevant counterterms. We shall turn to the analysis of $\Gamma$ in the next section, where it is shown that it is either finite or logarithmically divergent, which is indistinguishable from finite behaviour in the power counting.

\subsection{Logarithmic Divergences and Anomalies}

We have seen in Sec. 3.1 that a zero coefficient in front of one of the unknowns of the descent equations leads to a breakdown of the recursion and generically 
leaves behind an unresolved remainder, $\mathcal{H}_{\text {rem }}$. We shall discuss the meaning of $\mathcal{H}_{\text {rem }}$ now. In particular, we will show that it leads to a logarithmic divergence of $\Gamma$ and to a conformal anomaly in the boundary field theory. If we find that $\mathcal{H}_{\text {rem }}=0$ despite the breakdown, as it happens at level 0 in the case of the GPPZ flow potential, then there is no contribution to the anomaly.

The argument continues with the analysis of the Hamiltonian constraint, which, by solving the descent equations up to the breakdown, has been reduced to

$$
\mathcal{H}_{\text {rem }}+\mathcal{H}_{\Gamma}=0 \text {. }
$$

Moreover, we can express $\mathcal{H}_{\Gamma}$ to leading order as

$$
\begin{aligned}
\mathcal{H}_{\Gamma} & =8 q_{\Gamma}^{i j} q_{[0] i j}-\frac{8}{d-1} q_{\Gamma} q_{[0]}+\pi_{\Gamma} \pi_{[0]}+\cdots \\
& =-\frac{4 U}{d-1} q_{\Gamma}+U^{\prime} \pi_{\Gamma}+\cdots \\
& =\frac{1}{l}\left[\frac{2 g_{i j}}{\sqrt{g}} \frac{\delta \Gamma}{\delta g_{i j}}+\left(-\frac{d}{2}+\lambda\right) \phi \frac{1}{\sqrt{g}} \frac{\delta \Gamma}{\delta \phi}\right]+\cdots
\end{aligned}
$$

Here, we have used the results for $U$ from the previous section, and the ellipses stand for terms that are irrelevant in the $\rho \rightarrow \infty$ limit. Defining (remember $\Delta=d / 2+\lambda)$

$$
\mathcal{A}=\frac{2 g_{i j}}{\sqrt{g}} \frac{\delta \Gamma}{\delta g_{i j}}+(\Delta-d) \phi \frac{1}{\sqrt{g}} \frac{\delta \Gamma}{\delta \phi},
$$

we find from eqns. (40) and (41)

$$
\mathcal{A}=-l \mathcal{H}_{\text {rem }}+\cdots
$$

The quantity $\mathcal{A}$ is what dBVV call the conformal anomaly of the boundary field theory. This identification would follow from eqn. (42), if $\Gamma$ were the generating functional of the boundary field theory. Formally, $\mathcal{A}$ has the form of an anomaly, as can be seen in the particular cases. However, this interpretation is not correct. First, $\Gamma$ contains logarithmic divergences and is therefore not the generating functional of the boundary field theory. Second, $\mathcal{A}$ scales like $\mathrm{e}^{-d \rho / l}$ for $\rho \rightarrow \infty$, i.e., it has no finite limit, and, third, the boundary data $g_{i j}$ and $\phi$ are not the (renormalized) sources of the boundary field theory. We shall derive below that the true anomaly is obtained from $\mathcal{A}$ by a simple rescaling and taking the $\rho \rightarrow \infty$ limit. However, it is important to notice that the boundary integral of $\mathcal{A}$ is finite for $\rho \rightarrow \infty$ and has the form of an integrated anomaly, i.e., it is conformally invariant.

But first, we shall evaluate the logarithmic divergence of $\Gamma$. The argument uses the diffeomorphism invariance of $\Gamma$ and is similar to the arguments used in the derivation of the conformal anomaly by Henningson and Skenderis [8]. $\Gamma$ is a functional of the boundary data $g_{i j}$ and $\phi$ and depends explicitly on the value 
of the cut-off, $\rho$. We shall write it as $\Gamma\left[g_{i j}(x, \rho), \phi(x, \rho) ; \rho\right]$. By construction, $\Gamma$ is invariant under bulk diffeomorphisms,

$$
\Gamma\left[g_{i j}(x, \rho), \phi(x, \rho) ; \rho\right]=\Gamma\left[g_{i j}^{\prime}\left(x^{\prime}, \rho^{\prime}\right), \phi^{\prime}\left(x^{\prime}, \rho^{\prime}\right) ; \rho^{\prime}\right]
$$

In particular, under the following change of variables,

$$
x^{\prime}=x, \quad r^{\prime}=r(1+\sigma),
$$

the variation of $\Gamma$ becomes to first order

$$
\begin{aligned}
& \Gamma\left[g_{i j}\left(x, \rho^{\prime}\right), \phi\left(x, \rho^{\prime}\right) ; \rho^{\prime}\right]-\Gamma {\left[g_{i j}(x, \rho), \phi(x, \rho) ; \rho\right] } \\
&=\rho \sigma \int d^{d} x\left[\partial_{\rho} g_{i j} \frac{\delta \Gamma}{\delta g_{i j}}+\partial_{\rho} \phi \frac{\delta \Gamma}{\delta \phi}\right]+\mathcal{O}\left(\sigma^{2}\right) .
\end{aligned}
$$

Hence, after expressing $\partial_{\rho} g_{i j}$ and $\partial_{\rho} \phi$ to leading order, we find

$$
\partial_{\rho} \Gamma=\frac{1}{l} \int d^{d} x \sqrt{g} \mathcal{A}+\cdots
$$

where the boundary values $g_{i j}$ and $\phi$ are kept fixed when differentiating with respect to $\rho$ on the left hand side. Since the integral on the right hand side of eqn. (47) is finite for $\rho \rightarrow \infty$, this equation implies

$$
\Gamma=\frac{\rho}{l} \int d^{d} x \sqrt{g} \mathcal{A}+\text { finite terms }
$$

which is the logarithmic divergence of $\Gamma$. Eqn. (48) proves that $\Gamma$ does not contain power divergent terms. In other words, we have found all power divergent terms of the on-shell action by the analysis in Sec. 3.1.

It is interesting to ask why a breakdown of the descent equations is related to a logarithmic divergence. In the standard method, one solves the equations of motion in form of an asymptotic series. It is well known that, for certain powers of the leading behaviour, the series form of the solution will "break down", and one requires logarithms in order to continue the recursion. In the HJ method, the descent equations determine the momenta as a series of terms that locally depend on the fields, which, by virtue of the hamilton equations of motion, corresponds to the leading asymptotic series solution. Hence, the descent equations break exactly at the point, where the asymptotic series in the standard method would require a logarithm, and this translates into a logarithmically divergent term in the on-shell action.

Having found the logarithmic divergence, we define the renormalized on-shell action as

$$
S_{\text {ren }}=\lim _{\rho \rightarrow \infty}\left(\Gamma+\rho \int d^{d} x \sqrt{g} \mathcal{H}_{\mathrm{rem}}\right) .
$$


Since $\mathcal{A}$ has the form of a conformal anomaly, but vanishes for $\rho \rightarrow \infty$, it is natural to define a finite, rescaled quantity (cf. [20]),

$$
\hat{\mathcal{A}}=\lim _{\rho \rightarrow \infty}\left(\mathrm{e}^{d \rho / l} \mathcal{A}\right)
$$

$\hat{\mathcal{A}}$ is formally identical to $\mathcal{A}$ with $g_{i j}$ and $\phi$ replaced by $\hat{g}_{i j}$ and $\hat{\phi}$ (the coefficients of the leading terms in the asymptotic expansions), respectively. Then, the expression

$$
\int d^{d} x \sqrt{\hat{g}} \hat{\mathcal{A}}=\lim _{\rho \rightarrow \infty} \int d^{d} x \sqrt{g} \mathcal{A}
$$

is conformally invariant, and we confirm that $\hat{\mathcal{A}}$ is the conformal anomaly of the boundary field theory, ${ }^{7}$

$$
\begin{aligned}
\langle T\rangle-(\Delta-d) \hat{\phi}\langle\mathcal{O}\rangle & =\left[\frac{2 \hat{g}_{i j}}{\sqrt{\hat{g}}} \frac{\delta}{\delta \hat{g}_{i j}}+(\Delta-d) \hat{\phi} \frac{1}{\sqrt{\hat{g}}} \frac{\delta}{\delta \hat{\phi}}\right] S_{\text {ren }} \\
& =\lim _{\rho \rightarrow \infty}\left\{\mathrm{e}^{d \rho / l}\left[\frac{2 g_{i j}}{\sqrt{g}} \frac{\delta}{\delta g_{i j}}+(\Delta-d) \phi \frac{1}{\sqrt{g}} \frac{\delta}{\delta \phi}\right] \Gamma\right\} \\
& =\lim _{\rho \rightarrow \infty}\left(\mathrm{e}^{d \rho / l} \mathcal{A}\right)=\hat{\mathcal{A}} .
\end{aligned}
$$

In passing from the first to the second line we have used the fact that the integrated anomaly is conformally invariant.

\subsection{Special Case $\lambda=0$}

There is one logarithmic divergence that has not been addressed so far and which occurs in the case $\lambda=0$. In this case, no breakdown of the level 0 descent equation occurs, although the boundary integral of $\phi^{2}$ is finite. Hence, this divergence must be derived in a different fashion. This was considered also in [20], but the argument there is not complete.

In the case $\lambda=0$, the asymptotic behaviour of the scalar field is not given by the generic expression (3), but follows

$$
\phi(x, r)=\mathrm{e}^{-d r /(2 l)}\left[\frac{r}{l} \hat{\phi}(x)+\check{\phi}(x)+\cdots\right] .
$$

Thus,

$$
\partial_{r} \phi=-\frac{d}{2 l} \phi+\frac{1}{l} \mathrm{e}^{-d r /(2 l)} \hat{\phi}+\cdots
$$

$\overline{7 \text { We take }}-\hat{\phi}$ as the source coupling to the operator $\mathcal{O}$. The minus sign differs from the usual picture in the literature, but emerges from an improved correspondence formula [33]. Moreover, our energy momentum tensor differs by a minus sign from the convention of BFS. 
but we have also

$$
\partial_{r} \phi=\pi=U^{\prime}+\pi_{\Gamma}+\cdots .
$$

Comparing eqns. (54) and (55) and using eqns. (13) and (21), we can read off

$$
\pi_{\Gamma}=\frac{1}{l} \mathrm{e}^{-d r /(2 l)} \hat{\phi}+\cdots,
$$

where the ellipses stand for terms that are exponentially suppressed compared to the one written. In order to find the logarithmic divergence in terms of the fields living at the cut-off, write

$$
\frac{1}{\sqrt{g}} \frac{\delta \Gamma}{\delta \phi}=\pi_{\Gamma} \sim \frac{1}{\rho} \phi
$$

from which follows that

$$
\Gamma \sim \frac{1}{2 \rho} \int d^{d} x \sqrt{g} \phi^{2}
$$

in addition to any logarithmically divergent terms discussed in Sec. 3.2, which might arise for even $d$. Hence, in the case $\lambda=0$, the renormalized on-shell action is defined as

$$
S_{\text {ren }}=\lim _{\rho \rightarrow \infty}\left(\Gamma+\rho \int d^{d} x \sqrt{g} \mathcal{H}_{\mathrm{rem}}-\frac{1}{2 \rho} \int d^{d} x \sqrt{g} \phi^{2}\right) .
$$

The other special feature of this case is the calculation of the anomaly. As in Sec. 3.2, we can use the fact that, by solving the descent equations, the hamiltonian constraint has been reduced to eqn. (40), but $\mathcal{H}_{\Gamma}$ now has the form

$$
\mathcal{H}_{\Gamma}=8 q_{\Gamma}^{i j} q_{[0] i j}-\frac{8}{d-1} q_{\Gamma} q_{[0]}+\pi_{\Gamma} \pi_{[0]}+\frac{1}{2} \pi_{\Gamma}^{2}+\cdots .
$$

We have to include the term $\pi_{\Gamma}^{2}$, because in the power counting it has the same strength as the other terms. This yields

$$
\begin{aligned}
\langle T\rangle+\frac{d}{2} \hat{\phi}\langle\mathcal{O}\rangle & =\left[\frac{2 \hat{g}_{i j}}{\sqrt{\hat{g}}} \frac{\delta}{\delta \hat{g}_{i j}}-\frac{d}{2} \hat{\phi} \frac{1}{\sqrt{\hat{g}}} \frac{\delta}{\delta \hat{\phi}}\right] S_{\text {ren }} \\
& =\lim _{\rho \rightarrow \infty}\left\{\mathrm{e}^{d \rho / l}\left[\frac{2 g_{i j}}{\sqrt{g}} \frac{\delta}{\delta g_{i j}}-\frac{d}{2} \phi \frac{1}{\sqrt{g}} \frac{\delta}{\delta \phi}\right] \Gamma\right\} \\
& =\lim _{\rho \rightarrow \infty}\left[\mathrm{e}^{d \rho / l}\left(\mathcal{A}-\frac{l}{2} \pi_{\Gamma}^{2}\right)\right]=\hat{\mathcal{A}}-\frac{1}{2 l} \hat{\phi}^{2} .
\end{aligned}
$$

As before, we have used the conformal invariance of all logarithmic counterterms. In eqn. (61), $\mathcal{A}$ is defined by eqn. (43) [eqn. (42) does not hold here], so that $\hat{\mathcal{A}}$ denotes the gravitational conformal anomaly for even $d$, and the $\hat{\phi}^{2}$ term is the matter conformal anomaly. 


\section{One-point Functions}

\subsection{Exact One-point Functions}

One of the main results of the standard method of holographic renormalization is that it yields formal expressions for exact one-point functions [16]. Exact one-point functions depend on finite sources and thus contain information about all higher point functions of the theory. It is therefore desirable to obtain the same results using the Hamilton-Jacobi approach. This is straightforward, given the renormalized on-shell action, eqn. (49) [or eqn. (59) in the case $\lambda=0]$, and we shall demonstrate the procedure by considering the scalar one-point function for the cases $d=4, \lambda=1$ and $\lambda=0, d$ arbitrary.

Generally, we obtain

$$
\begin{aligned}
\langle\mathcal{O}(x)\rangle & =-\frac{1}{\sqrt{\hat{g}}} \frac{\delta S_{\mathrm{ren}}}{\delta \hat{\phi}(x)} \\
& =-\lim _{\rho \rightarrow \infty}\left[\mathrm{e}^{(d / 2+\lambda) \rho / l} \frac{1}{\sqrt{g}} \frac{\delta}{\delta \phi(x, \rho)}\left(\Gamma+\rho \int d^{d} x \sqrt{g} \mathcal{H}_{\mathrm{rem}}\right)\right] \\
& =-\lim _{\rho \rightarrow \infty}\left[\mathrm{e}^{(d / 2+\lambda) \rho / l}\left(\pi_{\Gamma}+\rho \frac{\delta \mathcal{H}_{\mathrm{rem}}}{\delta \phi}\right)\right]
\end{aligned}
$$

but, in practise, it is more useful to substitute $\pi_{\Gamma}$ from the split form (7) of the hamilton equation of motion (A.14),

$$
\pi_{\Gamma}=\partial_{r} \phi-\pi_{[0]}-\cdots-\pi_{[2 n]}
$$

because at this stage $\pi_{\Gamma}$ is not known explicitly in terms of the source. Moreover, it is necessary to write down an asymptotic expansion for $\phi$ beyond leading order, namely of the form of eqn. (3). However, in contrast to the standard method, we do not have to solve for the subleading coefficients using the equations of motion, an ansatz with the subleading $f_{1}, f_{2}$, etc. undetermined is sufficient. In practise, finding a general ansatz asks for some care, because generic bulk interactions might generate all sorts of exponents in the subleading terms. However, for our purposes, we shall regard this step as done.

Specializing to $d=4$ and $\lambda=1$, the potential $V$ has the form

$$
V(\phi)=-\frac{3}{l^{2}}-\frac{3}{2 l^{2}} \phi^{2}+\frac{v_{3}}{6} \phi^{3}+\frac{v_{4}}{4 !} \phi^{4}+\cdots
$$

This example is a generalization of the potential generating the GPPZ flow [32]. According to the results of Sec. (3.1), the function $U(\phi)$ occuring in the 
leading counterterm has the form

$$
U(\phi)=-\frac{3}{2 l}-\frac{1}{2 l} \phi^{2}+\frac{l v_{3}}{6} \phi^{3}+\frac{u_{4}}{4 !} \phi^{4}+\cdots
$$

Remember that the coefficient $u_{4}$ remains undetermined. Similarly, we have for the functions $\Phi(\phi)$ and $M(\phi)$,

$$
\begin{aligned}
\Phi(\phi) & =-\frac{l}{8}+\frac{1}{2} \Phi_{2} \phi^{2}+\cdots, \\
M(\phi) & =m_{0}+\cdots,
\end{aligned}
$$

with $\Phi_{2}$ and $m_{0}$ undetermined. The relevant asymptotic expansion for $\phi$ is of the form

$$
\phi(x, r)=\mathrm{e}^{-r / l} \hat{\phi}+\mathrm{e}^{-2 r / l} \phi_{1}+\mathrm{e}^{-3 r / l} \phi_{2}+\frac{r}{l} \mathrm{e}^{-3 r / l} \psi_{2}+\cdots,
$$

while it is sufficient to use the leading term for the metric,

$$
g_{i j}(x, r)=\mathrm{e}^{2 r / l} \hat{g}_{i j}+\cdots
$$

The unresolved remainder from the descent equations is given by eqns. (28), (38) and (39),

$\mathcal{H}_{\mathrm{rem}}=-\frac{l^{2}}{16}\left(\frac{1}{3} R^{2}-R_{i j} R^{i j}\right)-\frac{1}{2} \nabla^{i} \phi \nabla_{i} \phi-\frac{1}{12} R \phi^{2}+\left(\frac{l^{2} v_{3}^{2}}{8}-\frac{1}{3 l^{2}}-\frac{v_{4}}{24}\right) \phi^{4}$.

Thus, after substituting everything into eqn. (62), one finds

$$
\begin{aligned}
\langle\mathcal{O}\rangle= & \lim _{\rho \rightarrow \infty}\left\{\mathrm{e}^{\rho / l}\left(\frac{1}{l} \phi_{1}+\frac{l v_{3}}{2} \hat{\phi}^{2}\right)+\rho\left[\frac{2}{l^{2}} \psi_{2}-\hat{\nabla}^{2} \hat{\phi}+\frac{\hat{R}}{6} \hat{\phi}+\left(\frac{v_{4}}{6}+\frac{4}{3 l^{2}}-\frac{l^{2} v_{3}^{2}}{2}\right) \hat{\phi}^{3}\right]\right. \\
& \left.+\frac{2}{l} \phi_{2}-\frac{1}{l} \psi_{2}+l v_{3} \hat{\phi} \phi_{1}+\frac{u_{4}}{6} \hat{\phi}^{3}-m_{0} \hat{\nabla}^{2} \hat{\phi}+\Phi_{2} \hat{R} \hat{\phi}\right\} .
\end{aligned}
$$

Notice that the leading divergent term, proportional to $\mathrm{e}^{2 \rho / l}$, has explicitly cancelled. Moreover, since we have removed all divergences from the on-shell action (as was proven in Sec. 3.2), the correlation functions must be finite. Hence, the remaining two divergent terms in eqn. (71) must vanish by construction. Thus, we obtain the coefficients $\phi_{1}$ and $\psi_{2}$ of the leading asymptotic solution,

$$
\phi_{1}=-\frac{l^{2} v_{3}}{2} \hat{\phi}^{2}, \quad \psi_{2}=\frac{l^{2}}{2}\left[\hat{\nabla}^{2} \hat{\phi}-\frac{\hat{R}}{6} \hat{\phi}-\left(\frac{v_{4}}{6}+\frac{4}{3 l^{2}}-\frac{l^{2} v_{3}^{2}}{2}\right) \hat{\phi}^{3}\right] .
$$


Substituting eqn. (72) into eqn. (71), we finally obtain

$$
\langle\mathcal{O}\rangle=\frac{2}{l} \phi_{2}-\left(m_{0}+\frac{l}{2}\right) \hat{\nabla}^{2} \hat{\phi}+\left(\Phi_{2}+\frac{l}{12}\right) \hat{R} \hat{\phi}+\left(\frac{u_{4}}{6}+\frac{l v_{4}}{12}+\frac{2}{3 l}-\frac{3 l^{2} v_{3}^{2}}{4}\right) \hat{\phi}^{3}
$$

where the non-local behaviour is encoded in the undetermined coefficient $\phi_{2}$ of the subleading series solution, which is obtained by imposing regularity of the field in the bulk. The arbitrary parameters $u_{4}, \Phi_{2}$ and $m_{0}$ represent the scheme dependence. In particular, there exists a renormalization scheme, in which $\phi_{2}$ alone represents the exact scalar one-point function.

This example underlines the reverse approach of the HJ method with respect to the standard method of holographic renormalization. While, in the latter, one first determines the sub-leading coefficients in the asymptotic expansion of the fields from the equations of motion and then proceeds to renormalize the on-shell action, in the former one first renormalizes the on-shell action and then obtains the sub-leading coefficients from the finiteness of the correlation functions. One could ask whether the subleading coefficients obtained in eqn. (72) agree with those calculated in the standard method, i.e., whether they are consistent with the equations of motion. In the present example they obviously do, as seen by comparison with [16], but the answer is affirmative in general, because the coefficients follow uniquely by the procedure given above.

The case $\lambda=0$ is even simpler. Since the renormalized on-shell action is given by eqn. (59), we obtain

$$
\begin{aligned}
\langle\mathcal{O}\rangle & =-\frac{1}{\sqrt{\hat{g}}} \frac{\delta S_{\text {ren }}}{\delta \hat{\phi}} \\
& =-\lim _{\rho \rightarrow \infty}\left[\mathrm{e}^{d \rho /(2 l)} \frac{\rho}{l} \frac{1}{\sqrt{g}} \frac{\delta}{\delta \phi}\left(\Gamma-\frac{1}{2 \rho} \int d^{d} x \sqrt{g} \phi^{2}\right)\right] \\
& =-\lim _{\rho \rightarrow \infty}\left[\mathrm{e}^{d \rho /(2 l)} \frac{\rho}{l}\left(\pi_{\Gamma}-\frac{1}{\rho} \phi\right)\right]=\frac{1}{l} \check{\phi} .
\end{aligned}
$$

In the second line we have used the fact that $\mathcal{H}_{\text {rem }}$ does not depend on $\phi$ in the present case, while the last result follows from eqns. (53) and (56). This result is in agreement with our expectation, viz., $\check{\phi}$ corresponds to the vev in the boundary field theory.

The same procedure can be used to calculate the exact one-point function for 
the energy momentum tensor, $\left\langle T_{i j}\right\rangle$. The general formula is (for $\lambda \neq 0$ )

$$
\begin{aligned}
\left\langle T_{i j}\right\rangle & =-\frac{2}{\sqrt{\hat{g}}} \frac{\delta S_{\mathrm{ren}}}{\delta \hat{g}^{i j}} \\
& =\lim _{\rho \rightarrow \infty}\left[\mathrm{e}^{(d-2) \rho / l}\left(-\frac{2}{\sqrt{g}} \frac{\delta}{\delta g^{i j}}\right)\left(\Gamma+\rho \int d^{d} x \sqrt{g} \mathcal{H}_{\mathrm{rem}}\right)\right] \\
& =\lim _{\rho \rightarrow \infty}\left[\mathrm{e}^{(d-2) \rho / l}\left(2 q_{\Gamma i j}-\frac{2 \rho}{\sqrt{g}} \frac{\delta}{\delta g^{i j}} \int d^{d} x \sqrt{g} \mathcal{H}_{\mathrm{rem}}\right)\right]
\end{aligned}
$$

For $d=4$, the full analysis is quite tiresome and involves also scheme dependent counterterms, which are quadratic in the boundary curvature. We shall not perform the explicit expansion, since, for the GPPZ and the Coulomb branch flows, the result is known [16]. We only point out that the trace of the energy momentum tensor one-point function is given by the anomaly formulae, eqns. (52) and (61), and its divergence satisfies the Ward identity

$$
\hat{\nabla}_{j}\left\langle T^{i j}\right\rangle+\langle\mathcal{O}\rangle \hat{\nabla}^{i} \hat{\phi}=0
$$

Eqn. (76) follows from the constraint (A.18) and the fact that all counterterms are diffeomorphism invariant, because they are expressed as covariant boundary integrals.

\subsection{One-point Functions in Holographic RG Flows}

The calculation of one-point functions in holographic RG flows, i.e. those with the sources set to their background values, is particularly simple. Holographic RG flows are solutions of the bulk field equations of the form

$$
\phi=\phi(r), \quad g_{i j}=\mathrm{e}^{2 A(r)} \eta_{i j}
$$

which are obtained by solving the first order equations $[34,35,36]$

$$
\partial_{r} \phi=W^{\prime}(\phi), \quad \partial_{r} A=-\frac{2}{d-1} W(\phi)
$$

if the potential $V$ can be written in terms of a function $W(\phi)$ as

$$
V(\phi)=-\frac{d}{d-1} W^{2}+\frac{1}{2}\left(W^{\prime}\right)^{2}
$$

In addition, for $r \rightarrow \infty$, the solution should approach a fixed point of $W$ with negative value, so that the bulk becomes asymptotically AdS.

It is obvious that eqn. (79) is identical to the level zero constraint, eqn. (11), when $W$ is substituted for $U$. Similarly, eqn. (78) are the Hamilton equations 
of motion, (A.13) and (A.14). This means that $W$ necessarily is a solution of the level zero descent equation, but in order to be used in the leading counterterm, it must have an expansion

$$
W(\phi)=-\frac{d-1}{2 l}+\frac{1}{2 l}\left(-\frac{d}{2}+\lambda\right) \phi^{2}+\cdots .
$$

In particular, the quadratic coefficient must be $-d / 2+\lambda$ with $\lambda>0 .{ }^{8}$ Let us assume for the moment that this is the case. Then, after identifying $W \equiv U$, the calculation of the scalar one-point function becomes trivial:

$$
\langle\mathcal{O}\rangle=-\lim _{\rho \rightarrow \infty}\left[\mathrm{e}^{(d / 2+\lambda) \rho / l}\left(\partial_{r} \phi-U^{\prime}+\rho \frac{\delta \mathcal{H}_{\mathrm{rem}}}{\delta \phi}\right)\right]=0
$$

The second equality holds because of eqn. (78) and because neither the higher order counterterms nor $\mathcal{H}_{\text {rem }}$ contribute in the Poincaré invariant background.

Similarly, for the energy momentum tensor one finds

$$
\left\langle T_{i j}\right\rangle=\lim _{\rho \rightarrow \infty}\left[\mathrm{e}^{(d-2) \rho / l}\left(-\frac{d-1}{2} \partial_{r} A-U\right) g_{i j}\right]=0 .
$$

Eqn. (81) explicitly shows that prepotentials $W$ with the expansion (80) are flows generated by the addition of a relevant operator to a CFT Lagrangian.

Notice also that the choice $U=W$ for the leading counterterm means that we fix the coefficients in $U$, which are left undetermined by a possible breakdown of the level zero descent equation. This corresponds to a supersymmetric renormalization scheme, which is supported by the vanishing of the energy momentum tensor (82) and of $S_{\text {ren }}$ for a bulk solution of the form (78).

The second possibility, which we shall now discuss, is that $W$ has the expansion

$$
W(\phi)=-\frac{d-1}{2 l}+\frac{1}{2 l}\left(-\frac{d}{2}-\lambda\right) \phi^{2}+\cdots .
$$

Here, $\lambda$ is again positive. In this case, the background scalar field has the asymptotic behaviour

$$
\phi(r)=\mathrm{e}^{-(d / 2+\lambda) \rho / l} \check{\phi}+\cdots,
$$

which is entirely sub-leading with respect to the generic behaviour. Then,

8 This is the only detail, which is not fixed by the level zero constraint, the asymptotically AdS metric and the existence of the fixed point. 
eqn. (62) yields

$$
\begin{aligned}
\langle\mathcal{O}(x)\rangle & =-\lim _{\rho \rightarrow \infty}\left[\mathrm{e}^{(d / 2+\lambda) \rho / l}\left(W^{\prime}-U^{\prime}+\rho \frac{\delta \mathcal{H}_{\mathrm{rem}}}{\delta \phi}\right)\right] \\
& =-\lim _{\rho \rightarrow \infty}\left[\mathrm{e}^{(d / 2+\lambda) \rho / l}\left(-\frac{2 \lambda}{l} \phi+O\left(\phi^{2}\right)\right)\right]=\frac{2 \lambda}{l} \check{\phi} .
\end{aligned}
$$

Notice that the terms in $\mathcal{H}_{\text {rem }}$, which do not contain the boundary curvature, stem from a breakdown of the level zero equation and involve $\phi$ with at least cubic power, and these terms vanish in the $\rho \rightarrow \infty$ limit due to the weak asymptotic behaviour of $\phi$. Eqn. (85) explicitly demonstrates that holographic RG flows with $W$ of the form (83) correspond to deformations of the boundary CFT by switching on a vev of the scalar operator. Also in this case the onepoint function of the energy momentum tensor vanishes. From eqn. (75) one finds

$$
\begin{aligned}
\left\langle T_{i j}\right\rangle & =\lim _{\rho \rightarrow \infty}\left\{\mathrm{e}^{(d-2) \rho / l}\left[(W-U) g_{i j}+2 \rho g_{i j} O\left(\phi^{3}\right)\right]\right\} \\
& =\lim _{\rho \rightarrow \infty}\left\{\mathrm{e}^{d \rho / l}\left[-\frac{\lambda}{l} \phi^{2} \hat{g}_{i j}+2 \rho \hat{g}_{i j} O\left(\phi^{3}\right)\right]\right\}=0,
\end{aligned}
$$

again due to the weak asymptotic behaviour of $\phi$.

Last, let us comment briefly on the case, where $W$ has the expansion (80) with $\lambda=0$. The asymptotic behaviour of the RG flow solution is given by eqn. (53) with $\hat{\phi}=0$. The scalar one-point function has been calculated in Sec. 4.1 and is given by eqn. (74). The energy momentum tensor again vanishes, since, in the additional counterterm, the scalar field is not strong enough to compensate the $1 / \rho$ factor.

\section{Contributions of the Vector Sector}

In the following we will add the vector sector of the theory to the gravity and scalar sectors discussed so far. We will show how the Ward identities previously derived are modified by the presence of interacting vector fields, including expected new contributions to the conformal anomaly, and discuss the additional current Ward identity associated with the $U(1)$ gauge invariance. In this way we provide an alternative derivation of the holographic chiral anomaly, already obtained in [16] by using the standard approach to holographic renormalization and in [24] by explicitly analyzing some dual supergravity solutions.

The physics of an RG flow in the presence of a residual $U(1) R$-symmetry is encoded in a bulk $d+1$ dimensional action, which contains a "massive" vector 
field $A_{\mu}$ together with a Stückelberg field $\alpha$, interacting with scalars and gravity (see $[23,16]$ ). Thus, our starting point is the gauge-invariant action (A.1). The Hamiltonian constraints associated with this are derived and summarized in the Appendix. Before we begin the analysis, let us say two words on the issue of the asymptotic scaling of the fields. When we include the new fields, the equations of motion become rather intricately coupled, and we have to make sure that interactions between the fields do not influence their leading asymptotic behaviour. As a simplification, one can consider gravity and vectors in some fixed scalar background. This is the assumption made by BFS, and we borrow their analysis of the leading behaviour of the vector sector fields. From [16], we see that

$$
\begin{aligned}
B_{i}(x, r) & =\hat{B}_{i}(x)+\cdots \\
\alpha(x, r) & =\hat{\alpha}(x)+\cdots \\
\alpha(x, r) & =\frac{r}{l} \hat{\alpha}(x)+\check{\alpha}(x)+\cdots
\end{aligned}
$$

for $r \rightarrow \infty$.

\subsection{Descent Equations and Conformal Anomaly}

As discussed in Sec. 3, in order to analyze the descent equations obtained from the constraint $\mathcal{H}=0$, it is convenient to group the terms of the local part of the on-shell action into different levels. The lowest possible counterterms of the vector sector are of level two and read

$$
S_{[2]}^{v}=\int d^{d} x \sqrt{g}\left[\frac{1}{2} N(\phi) g^{i j} B_{i} B_{j}+\frac{1}{2} P(\phi) g^{i j} \nabla_{i} \phi B_{j}\right] .
$$

After computing the momenta stemming from the sum of (8), (9), and (90), and inserting them into eqn. (A.17), one can see that the level zero equation is unchanged, and one can always solve the level two contributions by setting $P(\phi)=0$. This is justified, since we do not expect counterterms linear in $B_{i}$. In this way the analysis of the gravity-scalar sector remains unchanged with respect to Sec. 3.1. The new equation to be solved is

$$
\mathcal{H}_{[2]}^{v}=\left(\frac{U^{\prime} N^{\prime}}{2}-\frac{d-2}{d-1} U N+\frac{N^{2}}{2 K}-\frac{\mathcal{M}^{2}}{2}\right) B^{i} B_{i}=0 .
$$

We proceed in the usual way by further expanding $U, N$ and $\mathcal{M}^{2}$ in powers of $\phi$. Notice that, for the flows we are interested in, $K(\phi)=O(1)$ and $\mathcal{M}^{2}(\phi)=$ $O\left(\phi^{2}\right)$. Using the solution of $U$ obtained in Sec. 3.1, we first get

$$
N_{0}\left(\frac{N_{0}}{2 K_{0}}+\frac{d-2}{2 l}\right)=0
$$


Again we shall compare to pure AdS/CFT results in order to select one of the two possibilities in the above equation. Looking for instance in [37] we learn that one should have $N_{0}=0$ for asymptotically massless vector fields. Proceeding up to quadratic order in $\phi$, we unambiguously determine

$$
\begin{aligned}
& N_{1}=0 \\
& N_{2}=\frac{l}{\lambda-1} \mathcal{M}_{2}^{2} .
\end{aligned}
$$

A breakdown occurs, if $\lambda=1$, thus giving the contribution

$$
\mathcal{H}_{\mathrm{rem}}=\frac{1}{2} \mathcal{M}_{2}^{2} \phi^{2} B^{i} B_{i}+O\left(\phi^{3}, B^{4}\right) \quad(\lambda=1) .
$$

Following the general analysis of Sec. 3.2 this yields a logarithmic divergence of $\Gamma$ and a contribution to the conformal anomaly for scalar operators with $\lambda=1$.

Proceeding to the next levels increases the number of invariants very quickly, and a complete analysis already at level four would be extremely tedious. The gravity-scalar sector has been analysed in [20], and in the following we restrict to the vector sector in a fixed scalar background. A possible basis of independent invariants for the level four on-shell action is the following

$$
\begin{aligned}
& \left(B_{i} B^{i}\right)^{2}, \nabla^{i} B_{i} B_{j} B^{j}, B_{i} B_{j} \nabla^{i} B^{j}, F_{i j} F^{i j},\left(\nabla^{i} B_{i}\right)^{2} \\
& \nabla_{i} B_{j} \nabla^{j} B^{i}, \nabla_{i} B_{j} \nabla^{i} B^{j}, R_{i j} B^{i} B^{j}, \nabla^{i} B^{j} R_{i j} .
\end{aligned}
$$

It turns out that the resulting level four descent equations, which we do not write here, can be consistently solved setting to zero the coefficients of all the terms except for $F_{i j} F^{i j}$. This leads to the descent equation

$$
\mathcal{H}_{[4]}^{F}=\left(\frac{d-4}{4 l} G_{0}-\frac{1}{4} K_{0}\right) F_{i j} F^{i j}=0,
$$

where $G_{0}$ is the constant part of the coefficient of the $F_{i j} F^{i j}$ counterterm. The descent equation (97) breaks for $d=4$, leading to a logarithmic divergence of $\Gamma$ and to a contribution to the conformal anomaly.

Hence, we have found the following anomaly contributions from the vector sector, up to level four:

$$
\hat{\mathcal{A}}= \begin{cases}-\frac{1}{2} l \mathcal{M}_{2}^{2} \hat{\phi}^{2} \hat{B}_{i} \hat{B}^{i} & (\lambda=1), \\ \frac{1}{4} l K_{0} \hat{F}_{i j} \hat{F}^{i j} & (d=4) .\end{cases}
$$

They agree with the logarithmic counterterms of [16], and the $d=4$ contribution was determined also in $[38,22]$. 


\subsection{Conformal Ward Identity}

Let us show how the anomalous conformal Ward identity is affected by the presence of currents. First, notice that the level zero terms of the $E^{i}$ and $\pi_{\alpha}$ momenta vanish, because the vector part of the on-shell action starts at level two [cf. eqn. (90)]. Therefore, $\mathcal{H}_{\Gamma}$ is the same at leading order as in eqn. (41). This implies that exactly the same form of conformal Ward identity holds here, though with the additional anomaly contributions derived in Sec. 5.1, eqn. (98), namely

$$
\langle T\rangle-(\Delta-d) \hat{\phi}\langle\mathcal{O}\rangle=\hat{\mathcal{A}}
$$

This might seem slightly odd. However, it is consistent with the scaling dimensions assigned to the various fields, which are read as usual from the leading $r$ dependence of the asymptotic expansions. In particular, recall that we have the following behaviours near the boundary $(r \rightarrow \infty)$

$$
\phi \sim \hat{\phi} \mathrm{e}^{-(d-\Delta) r / l} \quad A_{i} \sim \hat{A}_{i} \quad \alpha \sim \hat{\alpha}
$$

This implies that the sources $\hat{A}_{i}$ and $\hat{\alpha}$ do not transform under Weyl rescaling, and eqn. (99) correctly reflects this fact. On the other hand, it is well known that a conserved current should have conformal dimension $\tilde{\Delta}=d-1$, and thus couple to a vector source of weight one. In the context of the AdS/CFT correspondence this is realized by using as source the (rescaled) vector field in a local Lorentz frame, $A_{a}=e_{a}^{i} A_{i}$ [37]. Hence, we should have used the frame fields $A_{a}$ and the vielbeins $e_{i}^{a}$ as "coordinates" in the Hamiltonian treatment of the system. This would complicate the analysis unnecessarily, but it is easy to correct eqn. (99) for this misuse. In fact, setting $S_{\text {ren }}\left[g_{i j}, B_{i}\right]=\tilde{S}_{\text {ren }}\left[e_{i}^{a}, B_{a}\right]$, a straightforward application of the chain rule gives

$$
\left\langle\tilde{T}_{i}^{a}\right\rangle=T_{i}^{a}+\hat{B}_{i}\left\langle J^{a}\right\rangle
$$

After tracing the above expression and inserting it into eqn. (99), we get

$$
\langle\tilde{T}\rangle-(\Delta-d) \hat{\phi}\langle\mathcal{O}\rangle-\hat{B}_{a}\left\langle J^{a}\right\rangle=\hat{\mathcal{A}}
$$

Anticipating the next section, we can use gauge invariance to rewrite eqn. (102) in its standard field theory form as

$$
\langle\tilde{T}\rangle-(\Delta-d) \hat{\phi}\langle\mathcal{O}\rangle-\hat{\alpha}\left\langle\mathcal{O}_{\alpha}\right\rangle-\hat{A}_{a}\left\langle J^{a}\right\rangle=\hat{\mathcal{A}}
$$

where we have used the freedom to add a total derivative to the anomaly. 


\subsection{Diffeomorphism and Gauge Ward Identities}

In this section we shall derive the Ward identities that follow from the hamiltonian constraints associated with bulk diffeomorphisms and gauge invariance. From the constraints (A.19) and (A.18) and the premises on the powerdivergent counterterms stated in Sec. 3.1 we get

$$
\begin{aligned}
\nabla_{i} E_{\Gamma}^{i}+\pi_{\alpha \Gamma} & =0 \\
2 \nabla_{j} q_{i \Gamma}^{j}-\nabla_{i} \phi \pi_{\Gamma}-F_{i j} E_{\Gamma}^{j}-B_{i} \pi_{\alpha \Gamma} & =0
\end{aligned}
$$

respectively. How these relations translate into Ward identities for the finite field theory quantities depends, as usual, on whether logarithmically divergent terms hidden in $\Gamma$ possibly violate the constraints. Let us assume here that all logarithmic counterterms are obtained by the breakdown of the descent equations as described in Sec. 5.1. These counterterms are, by construction, both covariant and gauge invariant. Thus, the relations (104) and (105) directly translate into the following Ward identities for gauge and diffeomorphism invariance, respectively,

$$
\begin{aligned}
\hat{\nabla}_{i}\left\langle J^{i}\right\rangle+\left\langle\mathcal{O}_{\alpha}\right\rangle & =0 \\
\hat{\nabla}^{j}\left\langle T_{i j}\right\rangle+\langle\mathcal{O}\rangle \nabla_{i} \hat{\phi}+\left\langle J^{j}\right\rangle \hat{F}_{i j}+\left\langle\mathcal{O}_{\alpha}\right\rangle\left(\hat{A}_{i}+\partial_{i} \hat{\alpha}\right) & =0 .
\end{aligned}
$$

The expression given by BFS as the diffeomorphism Ward identity (eqn. (4.19) in [16]) is obtained by substituting eqn. (106) into (107).

\subsection{Special Case}

As in Sec. (3.3), one has to be more careful, if the leading term of a field is logarithmic, as is the case for $\alpha$ in the Coulomb branch flow with a fixed scalar background [cf. eqn. (89)]. In fact the following analysis applies only if we consider gravity and vectors in a fixed scalar background (denoted with a bar). That is, we are neglecting the back reaction of a possible scalar source turned on. This would complicate the situation considerably, as can be seen by inserting the leading behaviour of $\alpha$ from eqn. (89) into the action: The source $\hat{\alpha}$ contributes to the asymptotic mass of the scalar $\phi$ violating the assumption that the leading order behaviour does not depend on interactions.

Let us discuss this special case and derive the anomalous Ward identities for the CB flow. Using eqns. (89) and (A.15) and following similar steps as in Sec. 3.3, we find

$$
\frac{1}{\sqrt{g}} \frac{\delta \Gamma}{\delta \alpha}=\pi_{\alpha \Gamma}=\frac{1}{l} \mathcal{M}^{2} \hat{\alpha} \sim \frac{1}{\rho} \mathcal{M}^{2} \alpha
$$


From this follows that

$$
\Gamma \sim \frac{\mathcal{M}_{2}^{2}}{4 \rho} \int d^{d} x \sqrt{g} \bar{\phi}^{2} \alpha^{2}
$$

in addition to the logarithmically divergent terms stemming from $\mathcal{H}_{\text {rem }}$. The renormalized on-shell action is given by

$$
S_{\text {ren }}=\lim _{\rho \rightarrow \infty}\left(\Gamma+\rho \int d^{d} x \sqrt{g} \mathcal{H}_{\text {rem }}-\frac{\mathcal{M}_{2}^{2}}{4 \rho} \int d^{d} x \sqrt{g} \bar{\phi}^{2} \alpha^{2}\right)
$$

Notice that, with the scalar background fixed, the additional counterterm in eqn. (59) is irrelevant, and we have not included it here.

The additional counterterm in eqn. (110) is not gauge invariant, which will violate the Ward identity (106). In fact, it follows straightforwardly from eqns. (104) and (108) that

$$
-\mathrm{e}^{-d \rho / l} \hat{\nabla}_{i}\left\langle J^{i}\right\rangle+\frac{1}{2 l} \mathcal{M}_{2}^{2} \bar{\phi}^{2} \hat{\alpha}=0
$$

Hence, we obtain

$$
\hat{\nabla}_{i}\left\langle J^{i}\right\rangle=\frac{1}{2 l} \mathcal{M}_{2}^{2} \check{\phi}^{2} \hat{\alpha}=\frac{1}{l} \hat{\alpha}
$$

where for the last equality we have substituted the specific values $\mathcal{M}_{2}^{2}=12$, $\check{\phi}=-1 / \sqrt{6}$ for the CB flow [16].

As an aside, we can also compute the vev of the operator dual to $\alpha$ for the CB flow,

$$
\begin{aligned}
\left\langle\mathcal{O}_{\alpha}\right\rangle & =-\frac{1}{\sqrt{\hat{g}}} \frac{\delta S_{\mathrm{ren}}}{\delta \hat{\alpha}} \\
& =-\lim _{\rho \rightarrow \infty}\left[\mathrm{e}^{d \rho / l} \frac{\rho}{l} \frac{1}{\sqrt{g}} \frac{\delta}{\delta \alpha}\left(\Gamma-\frac{\mathcal{M}_{2}^{2}}{4 \rho} \int d^{d} x \sqrt{g} \bar{\phi}^{2} \alpha^{2}\right)\right] \\
& =-\lim _{\rho \rightarrow \infty}\left[\mathrm{e}^{d \rho / l} \frac{\rho}{l}\left(\pi_{\alpha \Gamma}-\frac{\mathcal{M}_{2}^{2}}{2 \rho} \bar{\phi}^{2} \alpha\right)\right] \\
& =\lim _{\rho \rightarrow \infty}\left[\mathrm{e}^{d \rho / l} \frac{\mathcal{M}_{2}^{2}}{2 l} \bar{\phi}^{2} \check{\alpha}\right]=\frac{1}{2 l} \mathcal{M}_{2}^{2} \check{\phi}^{2} \check{\alpha}=\frac{1}{l} \check{\alpha} .
\end{aligned}
$$

The results (106) and (112) were derived in [16] within the standard approach to holographic renormalization and represent the holographic realization of the chiral symmetry breaking. As also noticed in $[39,24]$, it is clear that the spontaneous breaking of gauge symmetry in the CB case is essentially dual to a Higgs mechanism in the bulk, with $\bar{\phi}^{2}$ providing a mass for $B_{i}$ because of its weak asymptotic scaling. 
One might worry that the leading logarithmic behaviour will upset the diffeomorphism Ward identity (107), but the following calculation explicitly confirms it also in this case. From eqns. (105), (110) and (108) follows

$$
\begin{aligned}
\mathrm{e}^{-d \rho / l} \hat{\nabla}_{j} T_{i}^{j}+\frac{1}{4 \rho} \mathcal{M}_{2}^{2} \partial_{i}\left(\phi^{2} \alpha^{2}\right)+\mathrm{e}^{-d \rho / l} \partial_{i} \hat{\phi}\langle\mathcal{O}\rangle-\frac{1}{2 \rho} \mathcal{M}_{2}^{2} \phi \partial_{i} \phi \alpha^{2} & \\
& +\mathrm{e}^{-d \rho / l} \hat{F}_{i j}\left\langle J^{j}\right\rangle-\left(A_{i}+\partial_{i} \alpha\right) \frac{1}{2 l} \mathcal{M}_{2}^{2} \phi^{2} \hat{\alpha}=0 .
\end{aligned}
$$

Using the explicit results (112) and (113) this yields

$$
\begin{gathered}
\mathrm{e}^{-d \rho / l}\left[\hat{\nabla}_{j} T_{i}^{j}+\hat{\nabla}_{i} \hat{\phi}\langle\mathcal{O}\rangle+\hat{F}_{i j}\left\langle J^{j}\right\rangle-\hat{A}_{i} \hat{\nabla}_{j}\left\langle J^{j}\right\rangle\right]+\frac{1}{2 l} \mathcal{M}_{2}^{2} \phi^{2} \partial_{i} \alpha\left(\frac{l}{\rho} \alpha-\hat{\alpha}\right) \\
=\mathrm{e}^{-d \rho / l}\left[\hat{\nabla}_{j} T_{i}^{j}+\hat{\nabla}_{i} \hat{\phi}\langle\mathcal{O}\rangle+\hat{F}_{i j}\left\langle J^{j}\right\rangle-\hat{A}_{i} \hat{\nabla}_{j}\left\langle J^{j}\right\rangle+\partial_{i} \hat{\alpha}\left\langle\mathcal{O}_{\alpha}\right\rangle\right]=0 .
\end{gathered}
$$

\section{Acknowledgements}

We would like to thank M. Bianchi for stimulating discussions and K. Skenderis for clarifications regarding the standard method and for helping us with the revised version. D. M. is pleased to thank Università di Napoli "Federico II" and Università di Roma "Tor Vergata" for very kind hospitality during completion of this work, as well as generous financial support. D. M. acknowledges partial support by PPARC through SPG\#613. W. M. acknowledges the financial support provided through the European Community's Human Potential Programme under contract HPRN-CT-2000-00131 Quantum Spacetime, in which he is associated with INFN, LNF Frascati.

\section{A Hamilton-Jacobi Method}

In this appendix, we shall summarize the Hamilton-Jacobi approach for a system of Einstein gravity coupled to a scalar field and a vector field treated in the Stückelberg formalism. Although the summary is sufficiently self-contained for the purpose of this article, the reader is referred to standard texts, e.g., [40], for a detailed description of the method.

The Hamiltonian treatment of gravity involves the time-slicing formalism, which assumes that the bulk space-time manifold can be globally foliated into hypersurfaces specified by a "time" coordinate. ${ }^{9}$ Of course, with Euclidean

9 For other cases, such as Taub-NUT spacetimes (see [41,42] and references therein), the method must be extended. 
signature, there is no distinction between time- and space-like directions, but the method can be applied equally. As for notation, we adorn geometric bulk quantities with a tilde and leave those belonging to hypersurfaces unadorned. Greek indices, $\mu, \nu$, run from 0 to $d$, latin indices, $i, j$, from 1 to $d$, and the index $r$ is often used instead of the index 0. Our conventions for the curvature tensor are $R_{j k l}^{i}=\partial_{k} \Gamma_{j l}^{i}+\Gamma_{k m}^{i} \Gamma_{j l}^{m}-(k \leftrightarrow l), R_{i j}=R_{i k j}^{k}$.

For the applications of the present paper (see $[23,16]$ ), we shall consider the following action:

$$
\begin{aligned}
S= & \int d^{d+1} x \sqrt{\tilde{g}}\left[-\frac{1}{4} \tilde{R}+\frac{1}{2} \tilde{g}^{\mu \nu} \partial_{\mu} \phi \partial_{\nu} \phi+V(\phi)\right]+\frac{1}{2} \int d^{d} x \sqrt{g} H \\
& +\int d^{d+1} x \sqrt{\tilde{g}}\left[\frac{1}{4} K(\phi) F_{\mu \nu} F^{\mu \nu}+\frac{1}{2} \mathcal{M}^{2}(\phi) B_{\mu} B^{\mu}\right] .
\end{aligned}
$$

Here, $V(\phi)$ denotes a scalar potential, which has a local minimum (stable fixed point) at $\phi=0$. The second integral in eqn. (A.1) is the Gibbons-Hawking term, where $H$ is the trace of the second fundamental form of the boundary hypersurface. This term is included in order to remove second derivatives with respect to the boundary normal from the bulk integral. The vector sector contains a vector field $A_{\mu}$ and the Stückelberg field $\alpha$, with $B_{\mu}=A_{\mu}+\partial_{\mu} \alpha$ and $F_{\mu \nu}=\partial_{\mu} B_{\nu}-\partial_{\nu} B_{\mu}$.

Writing the bulk metric as

$$
\tilde{g}_{\mu \nu}=\left(\begin{array}{cc}
n_{i} n^{i}+n^{2} & n_{j} \\
n_{i} & g_{i j}
\end{array}\right),
$$

where $n$ and $n^{i}$ are called the lapse and shift functions, respectively, the second fundamental form of $r=$ const hypersurfaces is given by

$$
H_{i j}=-\frac{1}{2 n}\left(\partial_{r} g_{i j}-\nabla_{i} n_{j}-\nabla_{j} n_{i}\right)
$$

Using geometric identities, eqn. (A.1) can be identically re-written as ${ }^{10}$

$$
\begin{aligned}
S= & \int d^{d+1} x \sqrt{g} n\left[\frac{1}{4}\left(-R+H_{j}^{i} H_{i}^{j}-H^{2}\right)+\frac{1}{2} \tilde{g}^{\mu \nu} \partial_{\mu} \phi \partial_{\nu} \phi+V(\phi)\right] \\
& +\int d^{d+1} x \sqrt{g} n\left[\frac{1}{4} K(\phi) F_{\mu \nu} F^{\mu \nu}+\frac{1}{2} \mathcal{M}^{2}(\phi) B_{\mu} B^{\mu}\right] .
\end{aligned}
$$

The action (A.4) is invariant under bulk diffeomorphisms and gauge transformations, $\delta A_{\mu}=-\partial_{\mu} \delta \alpha$. We shall gauge fix the quantities

$$
n=1, \quad n^{i}=0, \quad A_{r}=0,
$$

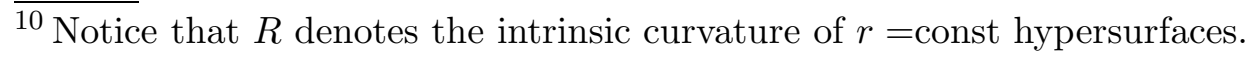


so that their corresponding equations of motion will enter as constraints into the Hamilton formalism,

$$
\begin{aligned}
-\frac{\delta S}{\delta n} & =\mathcal{H}=0 \\
\frac{\delta S}{\delta n^{i}} & =\mathcal{H}_{i}=0 \\
\frac{\delta S}{\delta A_{r}} & =\mathcal{G}=0
\end{aligned}
$$

where (in gauge fixed form)

$$
\begin{aligned}
\mathcal{H}= & \frac{1}{4}\left(R+H_{j}^{i} H_{i}^{j}-H^{2}\right)+\frac{1}{2}\left(\partial_{r} \phi\right)^{2}+\frac{1}{2} \mathcal{M}^{2} B_{r}^{2}+\frac{1}{2} K g^{i j} F_{r i} F_{r j} \\
& -\frac{1}{2} g^{i j} \partial_{i} \phi \partial_{j} \phi-\frac{1}{2} \mathcal{M}^{2} B_{i} B^{i}-\frac{1}{4} K F_{i j} F^{i j}-V \\
\mathcal{H}_{i}= & \frac{1}{2} \nabla_{j}\left(\delta_{i}^{j} H-H_{i}^{j}\right)-\partial_{r} \phi \partial_{i} \phi-\mathcal{M}^{2} B_{r} B_{i}-K g^{j k} F_{i j} F_{r k}
\end{aligned}
$$

and

$$
\mathcal{G}=\mathcal{M}^{2} B_{r}+\nabla^{i}\left(K F_{r i}\right)
$$

The gauge fixed action reads

$$
\begin{aligned}
S= & \int d^{d+1} x \sqrt{g}\left[-\frac{1}{4}\left(R-H_{j}^{i} H_{i}^{j}+H^{2}\right)+\frac{1}{2}\left(\partial_{r} \phi\right)^{2}+\frac{1}{2} g^{i j} \partial_{i} \phi \partial_{j} \phi+V\right. \\
& \left.+\frac{1}{2} \mathcal{M}^{2} B_{r}^{2}+\frac{1}{2} K g^{i j} F_{r i} F_{r j}+\frac{1}{2} \mathcal{M}^{2} B_{i} B^{i}+\frac{1}{4} K F_{i j} F^{i j}\right],
\end{aligned}
$$

where $H_{i j}=-\frac{1}{2} \partial_{r} g_{i j}, B_{r}=\partial_{r} \alpha$ and $F_{r i}=\partial_{r} A_{i}$.

This gauge fixed system can be treated in Hamilton language. The conjugate momenta are

$$
\begin{aligned}
q^{i j} & =\frac{1}{\sqrt{g}} \frac{\delta S}{\delta\left(\partial_{r} g_{i j}\right)}=\frac{1}{4}\left(g^{i j} H-H^{i j}\right), \\
\pi & =\frac{1}{\sqrt{g}} \frac{\delta S}{\delta\left(\partial_{r} \phi\right)}=\partial_{r} \phi \\
\pi_{\alpha} & =\frac{1}{\sqrt{g}} \frac{\delta S}{\delta\left(\partial_{r} \alpha\right)}=\mathcal{M}^{2} \partial_{r} \alpha, \\
E^{i} & =\frac{1}{\sqrt{g}} \frac{\delta S}{\delta\left(\partial_{r} A_{i}\right)}=K g^{i j} F_{r j} .
\end{aligned}
$$

Expressed in terms of the momenta, the constraints (A.6), (A.7) and (A.8) 
become

$$
\begin{aligned}
\mathcal{H}= & 4 q_{j}^{i} q_{i}^{j}-\frac{4}{d-1} q^{2}+\frac{1}{2} \pi^{2}+\frac{1}{2 \mathcal{M}^{2}} \pi_{\alpha}^{2}+\frac{1}{2 K} E_{i} E^{i} \\
& +\frac{1}{4} R-\frac{1}{2} g^{i j} \partial_{i} \phi \partial_{j} \phi-V-\frac{1}{2} \mathcal{M}^{2} B_{i} B^{i}-\frac{1}{4} K F_{i j} F^{i j}=0, \\
\mathcal{H}_{i}= & 2 \nabla_{j} q_{i}^{j}-\pi \partial_{i} \phi-B_{i} \pi_{\alpha}-F_{i j} E^{j}=0, \\
\mathcal{G}= & \pi_{\alpha}+\nabla_{i} E^{i}=0 .
\end{aligned}
$$

It is easy to realize that $\mathcal{H}$ coincides with the canonical hamiltonian density.

The bulk theory is defined on a bulk spacetime with a boundary at $r=\rho$, where $\rho$ is a cut-off parameter. In the Hamilton-Jacobi formalism the momenta of the theory are obtained from the on-shell action $S$ as a functional of prescribed boundary data, $g_{i j}(x, \rho), \phi(x, \rho), \alpha(x, \rho)$ and $A_{i}(x, \rho)$, from

$$
\begin{aligned}
q^{i j} & =\frac{1}{\sqrt{g}} \frac{\delta S}{\delta g_{i j}}, \\
\pi & =\frac{1}{\sqrt{g}} \frac{\delta S}{\delta \phi}, \\
\pi_{\alpha} & =\frac{1}{\sqrt{g}} \frac{\delta S}{\delta \alpha}, \\
E^{i} & =\frac{1}{\sqrt{g}} \frac{\delta S}{\delta A_{i}},
\end{aligned}
$$

where the variation is with respect to the boundary data. Therefore equations (A.17 - A.19) become constraints to be satisfied by $S$.

\section{References}

[1] J. M. Maldacena, Adv. Theor. Math. Phys. 2, 231 (1998), hep-th/9711200.

[2] S. S. Gubser, I. R. Klebanov and A. M. Polyakov, Phys. Lett. B 428, 105 (1998), hep-th/9802109.

[3] E. Witten, Adv. Theor. Math. Phys. 2, 253 (1998), hep-th/9802150.

[4] E. D'Hoker and D. Z. Freedman, hep-th/0201253.

[5] W. Mück and K. S. Viswanathan, Phys. Rev. D 58, 041901 (1998), hep-th/9804035.

[6] D. Z. Freedman, S. D. Mathur, A. Matusis and L. Rastelli, Nucl. Phys. B 546, 96 (1999), hep-th/9804058.

[7] H. Liu and A. A. Tseytlin, Nucl. Phys. B 533, 88 (1998), hep-th/9804083.

[8] M. Henningson and K. Skenderis, J. High Energy Phys. 07, 023 (1998), hep-th/9806087. 
[9] J. D. Brown and J. W. York, Phys. Rev. D 47, 1407 (1993).

[10] B. Balasubramanian and P. Kraus, Commun. Math. Phys. 208, 413 (1999), hep-th/9902121.

[11] R. C. Myers, Phys. Rev. D 60, 046002 (1999), hep-th/9903203.

[12] R. Emparan, C. V. Johnson and R. C. Myers, Phys. Rev. D 60, 104001 (1999), hep-th/9903238.

[13] E. Witten, Adv. Theor. Math. Phys. 2, 505 (1998), hep-th/9803131.

[14] L. Cappiello and W. Mück, Phys. Lett. B 522, 139 (2001), hep-th/0107238.

[15] S. de Haro, K. Skenderis and S. N. Solodukhin, Commun. Math. Phys. 217, 595 (2000), hep-th/0002230.

[16] M. Bianchi, D. Z. Freedman and K. Skenderis, Nucl. Phys. B 631, 159 (2002), hep-th/0112119.

[17] K. Skenderis, hep-th/0209067.

[18] M. Bianchi, D. Z. Freedman and K. Skenderis, J. High Energy Phys. 08, 041 (2001), hep-th/0105276.

[19] J. de Boer, E. Verlinde and H. Verlinde, J. High Energy Phys. 08, 003 (2000), hep-th/9912012.

[20] J. Kalkkinen, D. Martelli and W. Mück, J. High Energy Phys. 04, 036 (2001), hep-th/0103111.

[21] S. Corley, Phys. Lett. B 484, 141 (2000), hep-th/0004030.

[22] J. Kalkkinen and D. Martelli, Nucl. Phys. B 596, 415 (2001), hep-th/0007234.

[23] M. Bianchi, O. DeWolfe, D. Z. Freedman and K. Pilch, J. High Energy Phys. 01, 021 (2001), hep-th/0009156.

[24] I. R. Klebanov, P. Ouyang and E. Witten, Phys. Rev. D 65, 105007 (2002), hep-th/0202056.

[25] M. Bertolini, P. Di Vecchia, M. Frau, A. Lerda and R. Marotta, Phys. Lett. B 540, 104 (2002), hep-th/0202195.

[26] C. Fefferman and C. R. Graham, in Elie Cartan et les Mathématiques d'aujour d'hui, Astérique, p. 95 (1985).

[27] K. Skenderis, private communication.

[28] J. Parry, D. S. Salopek and J. M. Stewart, Phys. Rev. D 49, 2872 (1994), gr-qc/9310020.

[29] B. Darian, Class. Quant. Grav. 15, 143 (1998), gr-qc/9707046.

[30] V. L. Campos, G. Ferretti, H. Larsson, D. Martelli and B. E. W. Nilsson, J. High Energy Phys. 0006, 023 (2000), hep-th/0003151.

[31] D. Martelli and A. Miemic, J. High Energy Phys. 04, 027 (2002), hep-th/0112150.

[32] L. Girardello, M. Petrini, M. Porrati and A. Zaffaroni, Nucl. Phys. B 569, 451 (2000), hep-th/9909047. 
[33] W. Mück, Phys. Lett. B 531, 301 (2002), hep-th/0201100.

[34] D. Z. Freedman, S. S. Gubser, K. Pilch and N. P. Warner, Adv. Theor. Math. Phys. 3, 363 (1999), hep-th/9904017.

[35] O. DeWolfe, D. Z. Freedman, S. S. Gubser and A. Karch, Phys. Rev. D 62, 046008 (2000), hep-th/9909134.

[36] K. Skenderis and P. K. Townsend, Phys. Lett. B 468, 46 (1999), hep-th/9909070.

[37] W. Mück and K. S. Viswanathan, Phys. Rev. D 58, 106006 (1998), hep-th/9805145.

[38] M. M. Taylor-Robinson, hep-th/0002125.

[39] A. Brandhuber and K. Sfetsos, J. High Energy Phys. 12, 14 (2000), hep-th/0010048.

[40] C. W. Misner, K. S. Thorne and J. A. Wheeler, Gravitation, Freeman, San Francisco (1973).

[41] S. W. Hawking and C. J. Hunter, Phys. Rev. D 59, 044025 (1999), hep-th/9808085.

[42] S. W. Hawking, C. J. Hunter and D. N. Page, Phys. Rev. D 59, 044033 (1999), hep-th/9809035. 